\title{
The recombination hot spot $\chi$ is a regulatory element that switches the polarity of DNA degradation by the RecBCD enzyme
}

\author{
Daniel G. Anderson ${ }^{1}$ and Stephen C. Kowalczykowski ${ }^{1-3}$ \\ ${ }^{1}$ Genetics Graduate Group, ${ }^{2}$ Sections of Microbiology and of Molecular and Cellular Biology, University of California at \\ Davis, Davis, California 95616-8665 USA
}

\begin{abstract}
Homologous recombination in Escherichia coli is stimulated at DNA sequences known as $\chi$ sites. Stimulation requires the multifunctional RecBCD enzyme, which is both a helicase and a $3^{\prime} \rightarrow 5^{\prime}$ exonuclease. Upon recognition of a properly oriented $\chi$ site, the $3^{\prime} \rightarrow 5^{\prime}$ exonuclease activity is attenuated. Here we show that in addition to attenuation of the $3^{\prime} \rightarrow 5^{\prime}$ exonuclease activity, recognition of $\chi$ by the RecBCD enzyme also up-regulates a nuclease activity of the opposite polarity, resulting in an enzyme that now preferentially degrades $5^{\prime} \rightarrow 3^{\prime}$. These results demonstrate that $\chi$ is a unique regulatory element that converts the antirecombinogenic form of the RecBCD enzyme into a recombinogenic form by causing two distinct enzymatic changes: attenuation of the $3^{\prime} \rightarrow 5^{\prime}$ nuclease activity, and up-regulation of the $5^{\prime} \rightarrow 3^{\prime}$ nuclease activity. The consequence of $\chi$ recognition is the production of a recombination intermediate possessing a 3 '-ssDNA overhang terminating at the $\chi$ sequence. This processing of a dsDNA end to a 3 '-ssDNA overhang parallels that which occurs during the initation of homologous recombination in other pathways in $E$. coli, and in other organisms such as the yeast Saccharomyces cerevisiae.
\end{abstract}

[Key Words: Recombination; RecBCD; $\chi$; nuclease; helicase; regulation]

Received December 4, 1996; revised version accepted January 30, 1997.

The RecBCD enzyme is essential for the main pathway of homologous recombination in Escherichia coli. This heterotrimeric enzyme is composed of products of the $r e c B$, recC, and $r e c D$ genes (for review, see Smith 1988; Kowalczykowski et al. 1994). Inactivation of either the $r e c B$ or $r e c C$ genes leads to a reduction of homologous recombination by up to 1000 -fold in vivo (HowardFlanders and Theriot 1966; Emmerson 1968).

The RecBCD enzyme possesses a number of different nucleolytic activities; it is an ATP-dependent exonuclease and an ATP-stimulated single-stranded DNA (ssDNA) endonuclease (Telander-Muskavitch and Linn 1981; Taylor 1988; Smith 1990; Kowalczykowski et al. 1994). In addition to these degradative properties, the RecBCD enzyme is a highly processive helicase, capable of unwinding up to 30,000 bp per binding event at rates of up to $1000 \mathrm{bp} / \mathrm{sec}$ (Roman and Kowalczykowski 1989a; Roman et al. 1992; Eggleston and Kowalczykowski 1993). The preferred DNA substrate for the RecBCD enzyme is a double-stranded DNA (dsDNA) end, to which it binds with high affinity and then pro-

${ }^{3}$ Corresponding author.

E-MAIL sckowalczykowski@ucdavis.edu; FAX (916) 752-5939. ceeds to unwind the DNA duplex while simultaneously degrading the DNA from this end (Telander-Muskavitch and Linn 1981; Taylor and Smith 1985). Degradation of the DNA is asymmetric, with the 3 '-terminal strand relative to the entry site of the RecBCD enzyme being degraded much more extensively than the 5'-terminal strand (Dixon and Kowalczykowski 1993).

Homologous recombination in wild-type Escherichia coli is stimulated at cis-acting DNA sequences known as $\chi$ sites (5'-GCTGGTGG-3') (Lam et al. 1974; Stahl et al. 1975; Smith et al. 1981). In vivo, these DNA elements stimulate homologous recombination 5 - to 10 -fold in a rec $B C D$-dependent manner. Stimulation by $\chi$ is polar, with maximum stimulation at $\chi$ and decaying downstream relative to the entry site of the RecBCD enzyme (Stahl et al. 1980; Ennis et al. 1987; Cheng and Smith 1989; Myers et al. 1995a,b). The polar nature of $\chi$ stimulation in vivo is a direct result of the dramatic changes in the RecBCD enzyme function that take place after $\chi$ recognition. When $\chi$ is recognized by a translocating RecBCD enzyme molecule, DNA degradation halts $\sim 4-5$ nucleotides upstream of the $\chi$ site, after which the enzyme continues to unwind the DNA with its $3^{\prime} \rightarrow 5^{\prime}$ exonuclease activity greatly attenuated (Ponticelli et al. 1985; Dixon and Kowalczykowski 1993; Taylor and 
Smith 1995). The continued unwinding of the dsDNA downstream of $\chi$ leads to production of a ssDNA template that can now be utilized by the homologous pairing and strand-exchange protein RecA (Dixon and Kowalczykowski 1991).

Perhaps the single most essential enzyme for homologous recombination in $E$. coli is the RecA protein. $\mathrm{Mu}-$ tation of the recA gene causes a $10^{4}$ - to $10^{5}$-fold reduction in homologous recombination (Clark and Margulies 1965). In vitro, this protein is able to promote pairing and exchange between homologous DNA substrates (for review, see Kowalczykowski and Eggleston 1994). One requirement for RecA protein-promoted pairing in vitro is that at least one of the DNA substrates must possess some ssDNA character. This is in contrast to the observation that the majority of recombination events which occur in vivo, and in particular those that are mediated by the RecBCD pathway, involve donor DNA substrates that are double-stranded (for review, see Kowalczykowski et al. 1994). The RecBCD enzyme acts to initiate homologous recombination by processing dsDNA into a ssDNA substrate suitable for RecA protein action (Taylor and Smith 1985; Roman and Kowalczykowski 1989b; Dixon and Kowalczykowski 1991).

Although the ability of $\chi$ to attenuate the $3^{\prime} \rightarrow 5^{\prime}$ exonuclease of the RecBCD enzyme has been demonstrated, the effect of $\chi$ recognition on the weaker $5^{\prime} \rightarrow 3^{\prime}$ exonuclease activity is unclear (Dixon and Kowalczykowski 1993). In this paper we directly demonstrate that recognition of $\chi$ results in the up-regulation of the $5^{\prime} \rightarrow 3^{\prime}$ exonuclease activity, in addition to the attenuation of the $3^{\prime} \rightarrow 5^{\prime}$ exonuclease activity. Thus, the consequence of $\chi$ recognition is the production and net preservation of a recombination intermediate possessing a 3 '-ssDNA overhang terminating at the $\chi$ sequence. This processing of a dsDNA end to a 3 '-ssDNA overhang parallels that which occurs in other pathways of homologous recombination in $E$. coli and in other organisms such as the yeast $S$. cerevisiae (for review, see Kowalczykowski et al. 1994).

\section{Results}

Recognition of $\chi$ leads to the production of $\chi$-specific DNA fragments derived from both strands of dsDNA

As mentioned previously, recognition of $\chi$ by a translocating RecBCD enzyme molecule leads to attenuation of $3^{\prime} \rightarrow 5^{\prime}$ exonuclease activity. The continued unwinding of the DNA after $\chi$ produces a downstream ssDNA fragment of a length corresponding to the distance between $\chi$ and the distal end of the DNA (Dixon and Kowalczykowski 1993). In addition, Dixon and Kowalczykowski (1995) observed that another 5 '-end-labeled DNA fragment was created, possibly corresponding to the region of DNA that is upstream of $\chi$ but derived from the opposite DNA strand. For clarity, we define the region between the $\chi$ site and the end of the DNA at which the RecBCD enzyme initiated unwinding to be the upstream region, and the DNA between the $\chi$ site and the opposite end of the DNA to be the downstream region (see Fig. 1). In addition, the strand of dsDNA that terminates $3^{\prime}$ at the entry point of the RecBCD enzyme is defined as the top strand. The DNA strand opposite the top strand is defined as the bottom strand. Thus, the unwinding and specific cleavage of DNA containing a $\chi$ site by the RecBCD enzyme has the potential to generate four distinct $\chi$-specific ssDNA fragments: top strand, upstream fragment; top strand, downstream fragment; bottom strand, upstream fragment; and bottom strand, downstream fragment. To establish whether the DNA fragment observed by Dixon and Kowalczykowski (1995) was $\chi$-specific and derived from the bottom strand, unwinding reactions using linear pBR322 $\chi^{+} \mathrm{F}$ (Fig. 2A,B) were compared to those using linear pBR322 $\chi^{+} \mathrm{E}$ (Fig. $2 \mathrm{C})$, both of which contain a single $\chi$ site at a different location in $\mathrm{pBR} 322$. If the production of this new $5^{\prime}$-endlabeled species is $\chi$-specific, then the size of this species should change as the location of $\chi$ is varied. In addition, these reactions were compared to unwinding reactions using linear pBR322, which contains no $\chi$ sites (Fig. 2D).

Figure $2 \mathrm{~A}$ shows the products of an unwinding reaction using linearized pBR322 $\chi^{+} \mathrm{F}$, which was $5^{\prime}$-end-labeled. The efficiency of $\chi$ recognition by the RecBCD enzyme is only $\sim 20 \%$ (Taylor et al. 1985; Dixon and
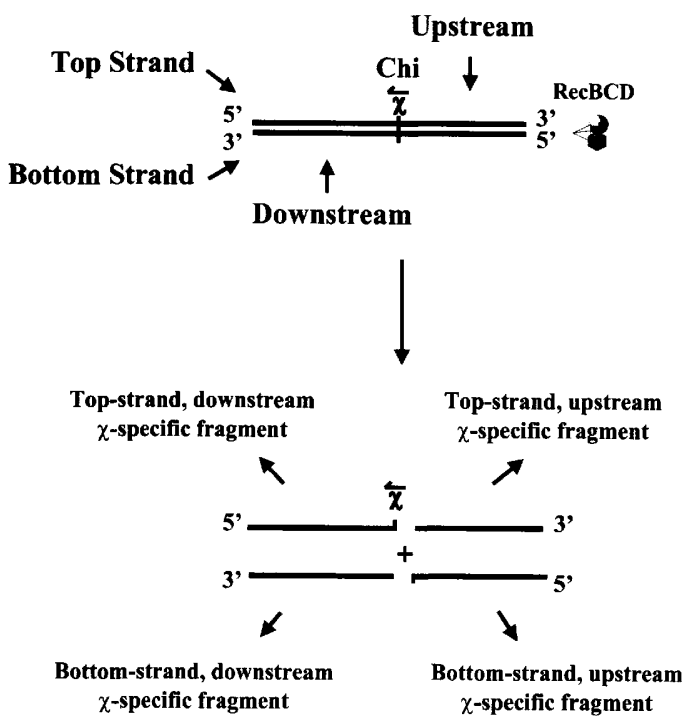

Figure 1. Possible $\chi$-specific fragments produced by the interaction of the RecBCD enzyme with $\chi$-containing DNA. The region of dsDNA between $\chi$ and the entry site for the RecBCD enzyme is referred to as the upstream region, and the region past $\chi$ to the opposite end of the DNA is the downstream region. The strand of DNA that terminates $3^{\prime}$ at the entry site of the RecBCD enzyme is termed the top-strand; the opposite strand is referred to as the bottom-strand. The combined nuclease and helicase action of the RecBCD enzyme on a $x$-containing dsDNA substrate has the potential to generate four $\chi$-specific ssDNA fragments: top-strand, upstream $\chi$-specific fragment; bottom-strand, upstream $\chi$-specific fragment; top-strand, downstream $\chi$-specific fragment; and bottom-strand, downstream $\chi$-specific fragment. 


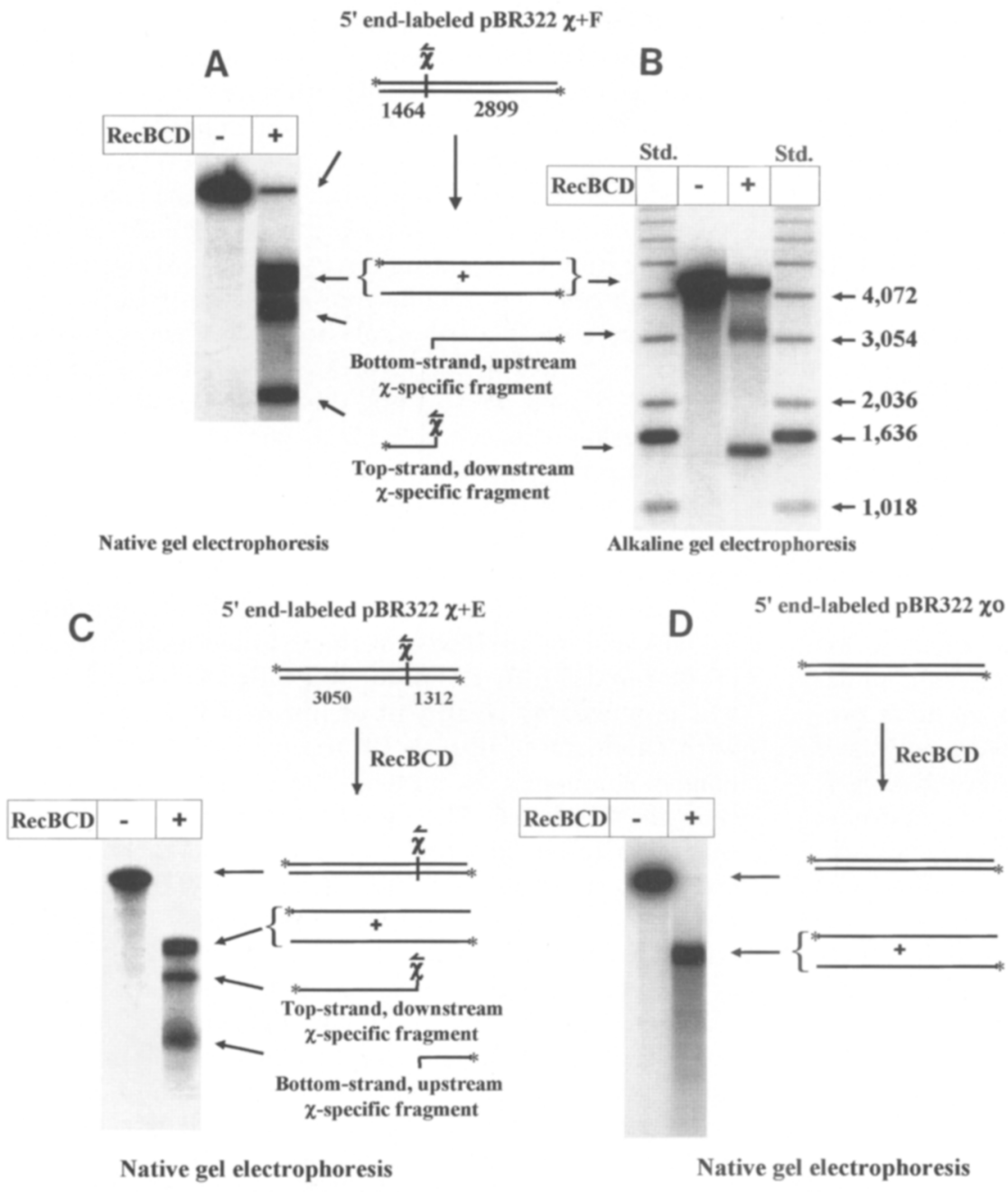

Figure 2. Production of a novel bottomstrand, upstream $\chi$-specific fragment by the RecBCD enzyme. The linear dsDNA substrates were created by restriction of pBR322 $\chi^{+} \mathrm{F}$ with HindIII $|A, B|$, pBR322 $\chi^{+} \mathrm{E}$ with $N d e I(C)$, and pBR322 $\chi^{\circ}$ with HindIII $(D)$. The arrow above the $\chi$ site indicates the direction that the RecBCD enzyme must travel to recognize $\chi$. The location of each $\chi$ site is given in nucleotides. The DNA was labeled at the $5^{\prime}$ end with ${ }^{32} \mathrm{P}$ and treated with one functional RecBCD enzyme per 20 dsDNA ends $(A, B, D)$ or one functional RecBCD enzyme per 5 dsDNA ends $(C)$. Full-length ssDNA (4363 nucleotides) and the 5 '-end-labeled, $\chi$-specific fragments are indicated. Reaction products were analyzed on a native $1 \%$ agarose gel $(A, C)$. Aliquots of reaction $(A)$ were loaded onto an alkaline agarose denaturing gel $(B)$.
Kowalczykowski 1993). Processing of dsDNA by the RecBCD enzyme without recognition of $x$ results in the production of full-length ssDNA because of the asymmetric degradation of DNA during unwinding (Dixon and Kowalczykowski 1993). Interaction of the RecBCD enzyme with $\chi$ apparently results in the formation of two $\chi$-specific fragments, the top strand, downstream $x$-specific fragment reported previously by Dixon and Kowalczykowski (1993), and another band, presumably corresponding to the bottom strand upstream of $\chi$. To determine the size of this putative upstream $\chi$-specific fragment, the reaction products were analyzed by denaturing alkaline agarose gel electrophoresis (Fig. 2B). Comparison of the size of this fragment with the $1-\mathrm{kb}$ ladder shows that cleavage occurred $\sim 300-500$ nucleotides downstream of the expected location on the basis of cleavage occurring precisely at the $\chi$ sequence. The precise size of the bottom-strand upstream $\chi$-specific fragment is dependent on the frequency of $5^{\prime} \rightarrow 3^{\prime}$ exonuclease cleavage, which is a function of free magnesium levels (Anderson et al. 1997).

To verify that the formation of the putative bottomstrand, upstream fragment is $\chi$-specific, a different $\chi$-containing DNA substrate was examined (Fig. 2C). The un- winding reaction shown in Figure $2 \mathrm{C}$ is the same as that in Figure 2A but uses a different starting dsDNA substrate: NdeI-linearized $5^{\prime}$-end-labeled pBR322 $\chi^{+}$E. Unwinding reactions using linear pBR322 $\chi^{+} \mathrm{E}$ DNA reveal the same sensitivity to reaction conditions that the pBR322 $\chi^{+}$F DNA showed (Anderson et al. 1997); most significantly, a novel 5 '-end-labeled ssDNA fragment corresponding to the region upstream of $\chi^{+} \mathrm{E}$ can be visualized (Fig. $2 \mathrm{C}$ ). For all of these reactions, the conditions used approximate intracellular free $\mathrm{Mg}^{2+}$ ion concentrations (Alatossava et al. 1985). Examination of unwinding reactions using wild-type pBR322, which does not contain a $\chi$ site, reveals that only full-length ssDNA is produced, further verifying that this novel species is $x$-specific (Fig. 2D).

As mentioned previously, the top-strand, downstream $x$-specific fragment results from degradation of the top DNA strand from the enzyme entry site to $\chi$, and attenuation of $3^{\prime} \rightarrow 5^{\prime}$ exonuclease at and beyond $\chi$ (see Dixon and Kowalczykowski 1993). The simplest explanation for generation of the novel bottom-strand, upstream $\chi$-specific fragment is a single cleavage event on the bottom strand opposite that of $\chi$. This would result in the formation of a bottom-strand, downstream $\chi$-specific 
fragment as well as a bottom-strand, upstream $\chi$-specific fragment. An alternative, but more complex explanation is that recognition of $\chi$ results in activation of the $5^{\prime} \rightarrow 3^{\prime}$ exonuclease of the RecBCD enzyme; this would result in destruction of a hypothetical bottom-strand, downstream $\chi$-specific fragment. To distinguish between these possibilities, $\chi$-containing DNAs were 3 '-end-labeled instead of 5 -end-labeled and treated with RecBCD enzyme.

\section{A bottom-strand, downstream $\chi$-specific fragment is not produced}

In contrast to the results obtained with $5^{\prime}$-end-labeled dsDNA, no bottom-strand $\chi$-specific bands are detected when $3^{\prime}$-end-labeled dsDNA is used (Fig. 3). Under no conditions is a $3^{\prime}$-end-labeled $\chi$-specific fragment corresponding to the region downstream from $\chi$ observed (Anderson et al. 1997). The conspicuous absence of this 3 '-end-labeled downstream $x$-specific fragment under conditions in which a $5^{\prime}$-end-labeled fragment is produced is not unique to pBR322 $\chi^{+} \mathrm{E}$ (Fig. 3A); analysis of the RecBCD enzyme-treated $3^{\prime}$-end-labeled pBR322 $\chi^{+} \mathrm{F}$, which could yield a shorter 3 '-end-labeled downstream $\chi$-specific fragment, reveals identical results (Fig. 3B). For both of these reactions, the only product of the RecBCD enzyme unwinding is full-length ssDNA. These results suggest that the hypothetical bottom-strand, downstream $\chi$-specific fragment was degraded, whereas the bottom-strand, upstream fragment was not.

Interaction of the RecBCD enzyme with $\chi$ up-regulates the $5^{\prime} \rightarrow 3^{\prime}$ exonuclease activity

The previous experiments showed that interaction of the RecBCD enzyme with $\chi$ leads to the production of a bot- tom-strand, upstream $\chi$-specific fragment, but not the corresponding bottom-strand, downstream fragment. There are three possible explanations for these observations, one of which is that the $5^{\prime} \rightarrow 3^{\prime}$ nonspecific nuclease activity is up-regulated after interaction with the $\chi$ site. The second possibility is that $\chi$ might be recognized in a "nonstandard" orientation by the RecBCD enzyme traveling from the opposite direction. A third alternative is that the production of bottom-strand, upstream $\chi$-specific fragments is attributable to DNA that was acted upon by two RecBCD enzymes, one of which degraded the DNA $3^{\prime} \rightarrow 5^{\prime}$ up to $\chi$ and then dissociated, followed by the action of a second RecBCD enzyme that degraded $3^{\prime} \rightarrow 5^{\prime}$ up to $x$ from the opposite direction. To distinguish between these alternatives, $\chi$-containing substrates were designed that limit the entry of the RecBCD enzyme to only one end.

The RecBCD enzyme can be prevented from entering a dsDNA end by a ssDNA overhang longer than 25 bases (Taylor and Smith 1985). Such a tailed DNA substrate was produced by treatment of linear pBR322 $\chi^{+} \mathrm{F}$ DNA with exonuclease III (Exo III) to create $5^{\prime}$ ssDNA overhangs $~ 90$ nucleotides in length at both ends /see Materials and Methods). The tailed DNA end upstream of $x$ was then removed using the restriction enzyme AseI, creating a substrate that is blocked at one of the two ends and contains a $\chi$ site oriented such that $\chi$ recognition will occur in the standard orientation for a RecBCD enzyme entering at the accessible end.

The products of DNA unwinding depend on the molar ratio of the RecBCD enzyme to DNA ends (Dixon and Kowalczykowski 1993). At subsaturating amounts of enzyme, the $\chi$-specific fragments and full-length ssDNA (when $\chi$ is not recognized) are produced (Fig. 4A); at saturating amounts of enzyme, half-length $5^{\prime}$-end-labeled ssDNA is produced by two RecBCD enzymes that meet
Figure 3. The bottom-strand, downstream $\chi$-specific fragment is not detected when $3^{\prime}$-end-labeled $\chi^{+}$DNA is used. The linear $\chi$-containing dsDNA substrates were created by cutting the plasmids pBR322 $\chi^{+} \mathrm{E}$ with NdeI $(A)$ and pBR322 $\chi^{+} \mathrm{F}$ with HindIII $(B)$. The DNA substrates each contain a $\chi$ site in a different location. The DNA was labeled at the $3^{\prime}$ end with ${ }^{32} \mathrm{P}$ and treated with one functional RecBCD enzyme per 20 dsDNA ends $(A, B)$. The reactions were analyzed on a $1 \%$ native agarose gel.

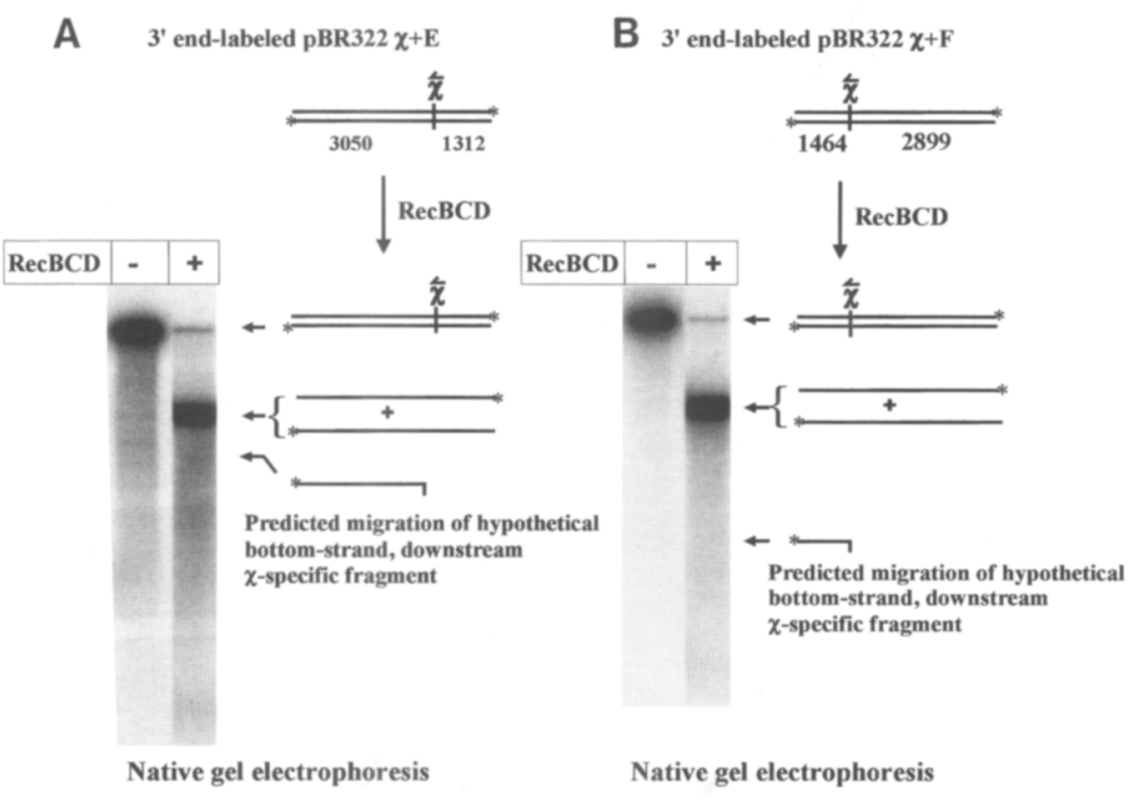


in the middle of the dsDNA substrate (Fig. 4B). If, however, entry at the DNA end downstream of $\chi$ is blocked by the presence of a ssDNA tail, treatment with a saturating RecBCD enzyme will produce the same DNA fragments that subsaturating amounts of enzyme do (Fig. $4 \mathrm{C})$. If the source of the bottom-strand, upstream $x$-specific fragment is up-regulation of the $5^{\prime} \rightarrow 3^{\prime}$ exonuclease activity upon $\chi$ recognition, then RecBCD enzyme action on this tailed DNA substrate should produce both bottom-strand, upstream and top-strand, downstream $\chi$-specific fragments. Finally, dsDNA that has tails at both ends should be completely resistant to the RecBCD enzyme (Fig. 4D).

Figure 5, lanes 7-12, shows the results of reactions with $\chi$-containing dsDNA substrates that were not treated with Exo III and, therefore, are accessible to the RecBCD enzyme from both dsDNA ends. Two different $5^{\prime}$-end-labeled $\chi$-containing substrates [pBR322 $\chi^{+} \mathrm{F}$ linearized by HindIII (lanes 10-12), and pBR322 $\chi^{+} \mathrm{F}$ cut with both HindIII and AseI (lanes 7-9)] were treated with varying amounts of the RecBCD enzyme. At subsaturating amounts of the RecBCD enzyme, unwinding leads to the production of full-length ssDNA, and both the upstream and downstream $5^{\prime}$-end-labeled $\chi$-specific fragments (lanes 8,11). The size of the bottom-strand, upstream $X$-specific fragment is smaller for the AseI-treated DNA substrate, as is the full-length ssDNA, because of cleavage of the region upstream from $\chi$ at the AseI site. The region downstream from $\chi$ was not altered by restriction of the $\mathrm{DNA}_{\text {; }}$ thus, the migration of the topstrand, downstream $\chi$-specific fragment is the same for these two substrates. At saturating enzyme concentrations (lanes 9,12), unwinding was initiated simultaneously from both ends, leading to the production of 5 '-end-labeled ssDNA that is approximately half of full length.

The $5^{\prime}$-end-labeled pBR322 $\chi^{+} \mathrm{F}$ DNA that had been treated with Exo III was also subjected to RecBCD enzyme treatment (lanes 1-6). This DNA has either the end downstream of $\chi$ or both ends blocked to the RecBCD enzyme access by the presence of a ssDNA tail, depending on whether or not the DNA was treated with AseI. The DNA that has a ssDNA overhang at both ends did not show any evidence of unwinding, even at the highest RecBCD enzyme concentrations (lanes 4-6). However, unwinding of the substrate that has a tail only at the end downstream of $\chi$ (lanes 1-3) produces both $\chi$-specific fragments and full-length ssDNA (owing to events where $\chi$ was not recognized) at both subsaturating and saturating RecBCD enzyme concentrations. The presence of this full-length ssDNA and the absence of half-length ssDNA at the highest RecBCD concentrations confirm that unwinding occurred from only one of the two ends. Most significantly, unwinding from the upstream end
A Sub saturating RecBCD neither end blocked by tail



Two Chi-specific fragments formed
B Saturating RecBCD neither end blocked by tail
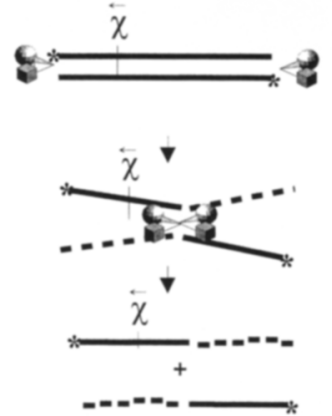

half-length ssDNA formed
C

Saturating RecBCD

down stream end blocked by tail

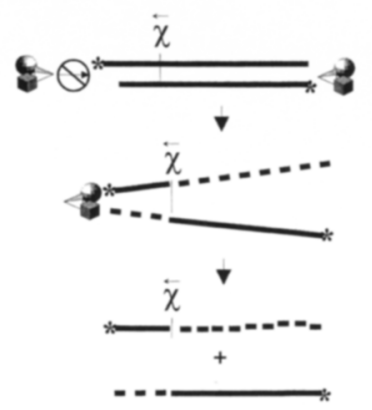

Two Chi-specific fragments formed
D Saturating RecBCD both ends blocked by tails

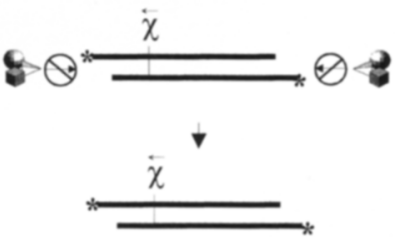

No Unwinding

Figure 4. Products expected when access of the RecBCD enzyme is restricted to one of the DNA ends. $(A)$ At subsaturating RecBCD enzyme concentrations (less than one RecBCD enzyme molecule per dsDNA end), RecBCD enzyme initiates unwinding and degradation from only one of the two ends. Upon recognition of $\chi$, the $3^{\prime} \rightarrow 5^{\prime}$ exonuclease activity is attenuated, whereas the $5^{\prime} \rightarrow 3^{\prime}$ exonuclease activity is up-regulated. Thus, $\chi$ recognition leads to the production of both top-strand, upstream and bottom-strand, downstream $\chi$-specific fragments. $(B)$ At saturating RecBCD enzyme concentrations (more than one RecBCD enzyme molecule per dsDNA end), simultaneous unwinding occurs from both ends of the dsDNA substrate. The $3^{\prime} \rightarrow 5^{\prime}$ exonuclease activity is synchronous; thus, the meeting of the two RecBCD enzyme molecules in the middle of a DNA substrate leads to production of $5^{\prime}$-end-labeled DNA that is approximately half of full length. In this case, $\chi$ is not recognized, as neither RecBCD enzyme encounters $\chi$ in the correct orientation. $(C)$ At saturating RecBCD enzyme concentrations, when entry at the downstream end is blocked by the presence of a ssDNA tail, RecBCD enzyme unwinding occurs only from the upstream end of the DNA. The products of this reaction are exactly the same as those produced under subsaturating conditions of the RecBCD enzyme on a dsDNA without a tail. However, the amount of $\chi$-specific fragment is doubled, because of the RecBCD enzyme approaching $\chi$ only from the productive orientation. $(D)$ At saturating RecBCD enzyme concentrations, when both of the available dsDNA ends are blocked by the presence of a ssDNA tail, the RecBCD enzyme is unable to unwind the DNA. 
leads to the production of both upstream and downstream $5^{\prime}$-end-labeled $x$-specific fragments. The formation of these products excludes the two hypotheses that required entry of the RecBCD enzyme from the end downstream of $\chi$. Therefore, the existence of both bottom-strand, upstream and top-strand, downstream $\chi$-specific fragments when $\chi$ is only approached from the upstream orientation is attributable to a switch in the polarity of nuclease activity at $\chi$.

To further verify that the presence of the bottomstrand, upstream $\chi$-specific fragment is attributable to an activation of nuclease activity at $\chi$, DNA was also blocked only at the end upstream of $\chi$ and subjected to RecBCD enzyme processing. Unwinding of DNA that possesses a ssDNA overhang upstream of $\chi$ behaves as if there were no $\chi$ sites present at all (data not shown). This is further proof that $\chi$ must be recognized in the standard orientation and that $\chi$-specific fragments are not being created following unwinding. Finally, time-course analysis of the production of $\chi$-specific fragments reveals that both top-strand, downstream and bottom-strand, upstream $\chi$-specific fragments are produced at the same rate (data not shown), illustrating further that $\chi$-specific fragments are a result of neither two sequential processing events nor post-unwinding recognition. Thus, the bottom-strand, upstream $\chi$-specific fragment is produced by a single RecBCD enzyme recognizing $\chi$ in the standard orientation. The production of this fragment is a direct consequence of a previously unknown facet of the interaction of RecBCD enzyme and $\chi$, namely that $\chi$ acts as a regulatory element to switch the polarity of DNA degradation by the RecBCD enzyme.

\section{Discussion}

The means by which the recombination hot spot $\chi$ acts to stimulate homologous recombination lies in the ability of $x$ to modify the nucleolytic properties of the RecBCD enzyme. One of the first biochemical observations of this process was the discovery that the RecBCD protein can produce a $\chi$-specific ssDNA fragment because of nicking in the vicinity of $\chi$ (Ponticelli et al. 1985; Taylor et al. 1985). Dixon and Kowalczykowski

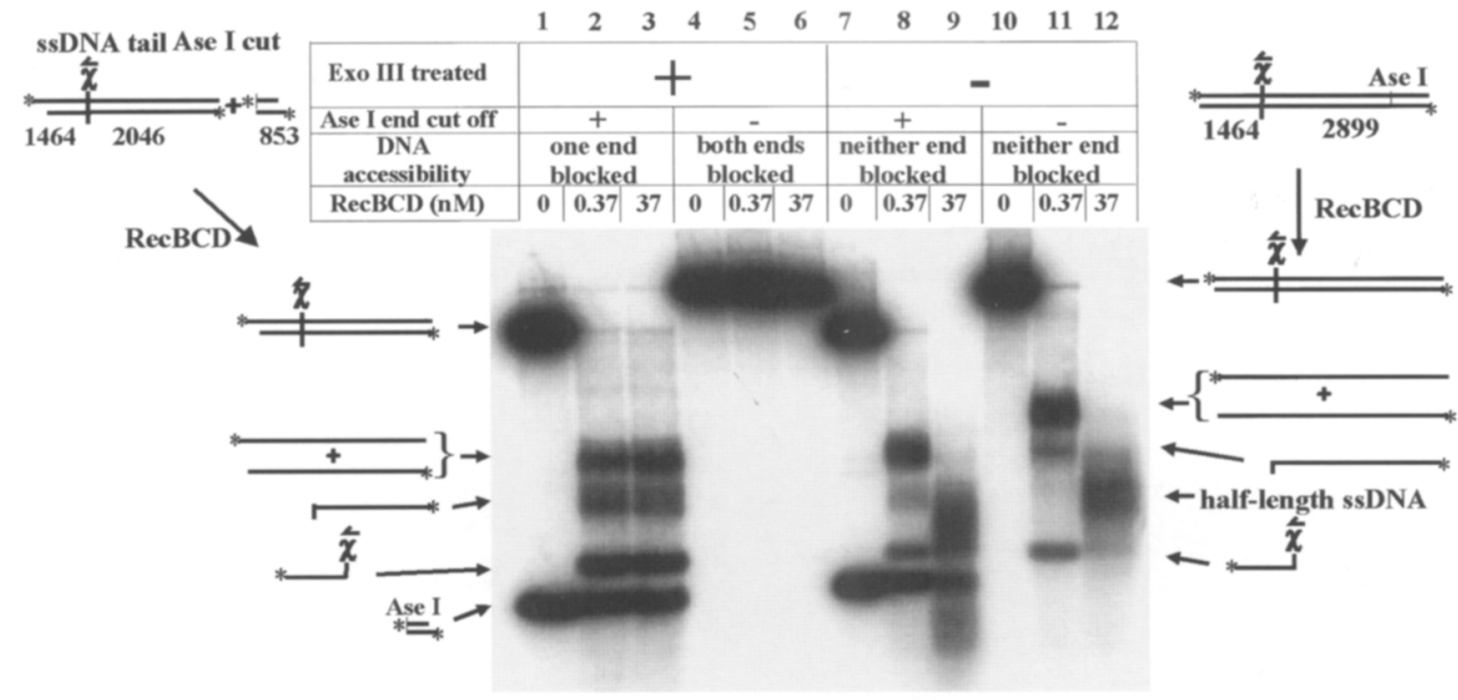

Figure 5. Blocking access of the RecBCD enzyme to DNA with ssDNA tails defines the orientation dependence of the novel $\chi$-specific fragment. Unwinding reactions were carried out under standard conditions using pBR $322 \chi^{+} \mathrm{F}$ dsDNA that was linearized by HindIII. Half of this DNA was treated with exonuclease III (lanes 1-6) to create a ssDNA tail of $\sim 90$ nucleotides in length. The tail at the upstream end was removed using AseI, and then the DNA substrates were 5 '-end-labeled. These DNA substrates were then treated with either subsaturating (one functional RecBCD enzyme per 17 dsDNA ends) or saturating amounts (six functional RecBCD enzyme molecules per 1 dsDNA end) of enzyme and incubated for $4 \mathrm{~min}$. The products were analyzed by native agarose gel electrophoresis. Lanes 1-3 show the products of an unwinding reaction using DNA that has the downstream end blocked by the presence of a ssDNA tail. Recognition of $\chi$ in the proper orientation leads to the production of both bottom-strand, upstream and top-strand, downstream $\chi$-specific fragments at all concentrations of RecBCD enzyme tested (see Fig. 4C for description of this case). Lanes 4-6 show an unwinding reaction in which both ends possess ssDNA tails; this DNA shows no unwinding at any RecBCD enzyme concentration, demonstrating the ssDNA tail as an effective block to RecBCD enzyme access (see Fig. 4D). Lanes 7-9 show an unwinding reaction using DNA that does not possess a ssDNA tail at either end; however, the upstream end was removed using AseI to give DNA that is the same length as the singly tailed DNA used in lanes 1-3. RecBCD enzyme treatment of this DNA leads to both upstream and downstream $\chi$-specific fragments at subsaturating RecBCD enzyme concentrations, and half-length ssDNA at saturating RecBCD protein (see Fig. 4, A and B, respectively). Lanes 10-12 show an unwinding reaction using DNA that does not possess a ssDNA tail at either end. Again, RecBCD enzyme treatment of this DNA leads to the production of both upstream and downstream $\chi$-specific fragments at subsaturating RecBCD protein concentrations; at saturating the RecBCD enzyme, the major product is half-length ssDNA. 
(1993) furthered this characterization by showing that the production of this $\chi$-specific fragment was a consequence of a more general phenomenon: the attenuation of the RecBCD enzyme's $3^{\prime} \rightarrow 5^{\prime}$ exonuclease activity upon interaction with $\chi$. However, the effect of $\chi$ recognition on the weaker $5^{\prime} \rightarrow 3^{\prime}$ exonuclease activity of the RecBCD protein remained undefined. Here we show that recognition of $\chi$ in the standard orientation by a translocating RecBCD molecule not only leads to the production of a bottom-strand, upstream $\chi$-specific fragment but also the degradation of the bottom-strand, downstream $\chi$-specific region. We demonstrated that this asymmetric production of $\chi$-specific fragments is a direct result of the unexpected up-regulation of the $5^{\prime} \rightarrow 3^{\prime}$ exonuclease activity upon recognition of $\chi$ (Fig. 6). This observation demonstrates that $\chi$ is a unique regulatory element, which acts to stimulate recombination by controlling the polarity of exonuclease degradation by the RecBCD enzyme.

The switch in the polarity of degradation at $\chi$ from $3^{\prime} \rightarrow 5^{\prime}$ to $5^{\prime} \rightarrow 3^{\prime}$ has important biological consequences for the initiation of homologous recombination. Athough the processive helicase action of the RecBCD enzyme leads to the complete unwinding of the small, pBR322-based plasmids used in vitro, the result of this polarity switch on longer DNA substrates (i.e., chromosomal or phage $\lambda$ DNA) would be a change from the production of DNA with $5^{\prime}$-overhanging ssDNA to DNA with $3^{\prime}$-overhanging ssDNA ends. The generation of 3 'ssDNA ends is critical with regard to RecA protein function. As mentioned previously, the RecA protein binds the ssDNA produced by RecBCD enzyme unwinding and then catalyzes the pairing of this DNA to a homologous region in its supercoiled counterpart, forming a recombination intermediate known as a joint molecule (Shibata et al. 1979; McEntee et al. 1980; Roman and Kowalczykowski 1989b; Dixon and Kowalczykowski 1991) (Fig. 6B). The binding of the RecA protein to ssDNA is cooperative and polar in nature, with extension of the RecA protein filament occurring $5^{\prime} \rightarrow 3^{\prime}$ (Register and Griffith 1985). This polarity is reflected in the bias shown in DNA strand invasion: $3^{\prime}$ Ends are approximately 10 -fold more invasive than $5^{\prime}$ ends in RecA protein-promoted joint molecule formation, a bias that is even more exaggerated in the presence of the singlestranded DNA binding (SSB) protein (Konforti and Davis $1987,1990)$. Thus, the switch in polarity of DNA degradation at $\chi$ from primarily $3^{\prime} \rightarrow 5^{\prime}$ to $5^{\prime} \rightarrow 3^{\prime}$ changes the RecBCD enzyme from a form that produces a DNA intermediate that has a poorly recombinogenic 5 '-overhanging ssDNA end to a modified form that produces a highly recombinogenic $3^{\prime}$-overhanging ssDNA end.

The helicase activity of RecBCD enzyme has long been postulated to produce a 3 '-ssDNA end that is used by the RecA protein for DNA strand invasion (see Smith 1988). The discovery that the $5^{\prime} \rightarrow 3^{\prime}$ exonuclease activity is up-regulated upon interaction with $\chi$ raises the question of what role this increase in degradation plays in homologous recombination. Perhaps the most reasonable explanation for the existence of this activity is that it removes a possible competitor for binding to the recombinogenic $3^{\prime}$ end of the $x$-specific ssDNA. Degradation of the DNA complementary to this $3^{\prime}$-ssDNA end would prevent reannealing and consequent inactivation of this invasive strand, making the ssDNA more accessible for RecA protein-promoted DNA strand invasion.

The hypothesis that initiation of homologous recombination from a dsDNA end requires the formation of a 3 '-ssDNA overhang is supported by suppressor analysis of $r e c B C D$ null mutations. The reduced recombination in these strains can be suppressed completely by the activation of another nuclease, the RecE protein (Barbour et al. 1970; Templin et al. 1972). In vitro, the RecE protein is a highly processive dsDNA-specific $5^{\prime} \rightarrow 3^{\prime}$ exonuclease that removes $10,000-20,000$ nucleotides from the $5^{\prime}$-terminal strand per binding event /Gillen et al.



Figure 6. Biochemical model for genetic recombination initiated from a dsDNA end. Details are discussed in the text. Initiation involves the resection of a dsDNA end to produce a 3 '-ssDNA overhang. $(A)$ This resection can occur through the action of the RecBCD enzyme and $\chi$, or the RecE protein, or the combined actions of the RecBC and RecJ proteins. (B) The 3' end of this ssDNA overhang invades a homolog in a reaction mediated by RecA and SSB proteins to form a D-loop. 
1977; Joseph and Kolodner 1983a,b; for review, see Kowalczykowski et al. 1994). Thus, the behavior of the RecE protein is very similar to the behavior of the RecBCD enzyme after it has interacted with a $x$ site: They both lead to the production of DNA with $3^{\prime}$ ssDNA tails (Fig. 6). The need to form a 3 '-ssDNA overhang is also supported by examination of homologous recombination in $r e c D$ null mutants. Cells that have functional $r e c B$ and $\operatorname{rec} C$ but nonfunctional $r e c D$ have approximately normal levels of recombination, but they show a genetic dependence on another nuclease, the RecJ protein (Lovett et al. 1988). In vitro, the RecBC protein (i.e., without the RecD subunit) is an active helicase with little or no measurable nuclease activity (Palas and Kushner 1990; Masterson et al. 1992; Korangy and Julin 1993, Anderson et al. 1997). The RecJ protein is a singlestrand specific exonuclease with a polarity that is $5^{\prime} \rightarrow 3^{\prime}$. It is likely then that in the absence of RecD subunit, the RecBC protein initiates unwinding at a dsDNA end and the RecJ protein resects the resultant 5 '-terminal ssDNA (Fig. 6); degradation by the RecJ protein of ssDNA that was formed by the RecBC protein was demonstrated in vivo (Rinken et al. 1992). Again, the consequence of this separated helicase/nuclease action is formation of $3^{\prime}$-overhanging ssDNA that could then be used by the RecA protein to promote DNA strand invasion. Thus, the processing of DNA during the initiation step of the RecBCD enzyme mediated recombination is the same as that occurring during initiation of homologous recombination in other pathways in E. coli and in $S$. cerevisiae: in all cases, the processed product has a $3^{\prime}$ ssDNA overhang.

As described above, processing by the RecBCD enzyme places $\chi$ at the $3^{\prime}$ end of the ssDNA tail. Analysis of DNA strand invasion reactions catalyzed by RecA and RecBCD proteins reveals that the formation of joint molecules using the $\chi$-containing $3^{\prime}$-ssDNA end is favored over non $x$-containing ssDNA (Dixon and Kowalczykowski 1991, 1995). Recent examination of this process has revealed that the $x$ sequence not only can act to directly stimulate RecA protein-promoted pairing (Tracy and Kowalczykowski 1996) but also to stimulate the loading of the RecA protein onto the $\chi$-containing ssDNA strand in a RecBCD enzyme-dependent process (D.G. Anderson and S.C. Kowalczykowski, in prep.). Thus, the ability of the RecBCD protein to translocate a $3^{\prime}$ end so that $\chi$ will be at the $3^{\prime}$ end further enhances the efficiency of joint molecule formation.

Although the specific modification of the RecBCD enzyme affected by $\chi$ recognition remains unclear, evidence suggests that interaction of the RecBCD protein with $\chi$ leads to the reversible inactivation/ejection of the RecD subunit (Thaler et al. 1988; Stahl et al. 1990; Dixon et al. 1994; Koppen et al. 1995; Myers et al. 1995a). As mentioned previously, the RecBC protein is a helicase but has little or no nuclease activity. The fact that the RecBCD enzyme after interaction with $\chi$ is not only a helicase, but also a $5^{\prime} \rightarrow 3^{\prime}$ exonuclease now raises the question of whether the RecD subunit is actually ejected at $\chi$. Both the $3^{\prime} \rightarrow 5^{\prime}$ exonuclease activity of RecBCD enzyme before $\chi$ and the $5^{\prime} \rightarrow 3^{\prime}$ exonuclease after $\chi$ require functional RecD protein, suggesting that the RecD subunit is directly responsible for one or both of these activities. Another formal possibility is that inactivation of the RecD subunit after recognition of $\chi$ activates a nuclease domain in either the RecB or RecC subunits, which is inactive in the RecBC enzyme. Although the biochemical basis of $\chi$ recognition remains a mystery, it is interesting to note that the RecD protein possesses a region of sequence similarity with $\mathrm{RecJ}$ and a number of other 5' $\rightarrow$ 3' specific DNA exonucleases (S.T. Lovett, pers. comm.).

The finding here that processing of a dsDNA break by the RecBCD enzyme and $\chi$ leads to the production of a 3 '-ssDNA overhang is significant in that it brings all the pathways of recombination in E. coli into conformity. These findings support a universal model for homologous recombination that involves initiation from a $3^{\prime}$ ssDNA overhang and that also pertains to replicative repair processes (Kowalczykowski et al. 1994; Kogoma 1996) (Fig. 6). In this model initiation occurs through resection of a dsDNA end to a $3^{\prime}$-overhang, which can then be used by the RecA protein to catalyze D-loop formation as described previously.

The idea that initiation of homologous recombination involves a $3^{\prime}$-ssDNA overhang is neither new (Resnick 1976; Szostak et al. 1983) nor unique to E. coli. Characterization of the processing of dsDNA breaks in the yeast $S$. cerevisiae has revealed that at both $\mathrm{HO}$ endonuclease-induced breaks (White and Haber 1990) and the ARG4 initiation site for meiotic gene conversion (Sun et al. 1991), the first processing event that occurs is the asymmetric resection of the DNA producing extensive, ssDNA tails with $3^{\prime}$ overhangs. We have shown that the RecBCD enzyme-dependent processing of dsDNA breaks, mediated by $\chi$, leads to the same end result, but focused at $\chi$. Thus, the processing of dsDNA breaks to form $3^{\prime}$-ssDNA overhangs may represent a universal aspect of the initiation of homologous recombination and recombinational DNA repair.

\section{Materials and methods}

Enzymes

The RecBCD enzyme was purified as described (Roman and Kowalczykowski 1989a). Protein concentration was determined using an extinction coefficient of $4.0 \times 10^{5} / \mathrm{M} / \mathrm{cm}$ at $280 \mathrm{~nm}$ (Roman and Kowalczykowski 1989a). The specific activity of the enzyme preparation was $5.4 \times 10^{4}$ units of nuclease or $1.1 \times 10^{4}$ units of helicase per milligram of protein. Nuclease units and helicase units were measured as described (Eichler and Lehman 1977; Roman and Kowalczykowski 1989a, respectively|. SSB protein was isolated from strain RLM727 and purified according to LeBowitz (1985). Protein concentration was determined using an extinction coefficient of $3.0 \times 10^{4} / \mathrm{M} / \mathrm{cm}$ at $280 \mathrm{~nm}$ (Ruyechan and Wetmur 1975). All restriction enzymes and DNA-modifying enzymes were purchased from New England Biolabs, Pharmacia LKB, Bethesda Research Laboratories, or Promega. The enzymes were used as specified by the vendors. 


\section{DNA substrates}

Plasmids pBR322 $\chi^{\circ}$ (wild type), pBR322 $\chi^{+} \mathrm{F}$, and pBR322 $\chi^{+} \mathrm{E}$ (Smith et al. 1981) were purified using alkaline lysis as described in Sambrook et al. (1989). The molar concentration of dsDNA in nucleotides was determined using an extinction coefficient of $6290 / \mathrm{M} / \mathrm{cm}$ at $260 \mathrm{~nm}$. Plasmid DNA was linearized with the appropriate restriction enzyme and then end-labeled at either the 3 ' end using the Klenow fragment of DNA polymerase I and appropriate $\left[\alpha^{-32} \mathrm{P}\right] \mathrm{dNTP}$ (NEN), or at the $5^{\prime}$ end by sequential reactions with shrimp alkaline phosphatase and $\mathrm{T} 4$ polynucleotide kinase in the presence of $\left[\gamma^{-32} \mathrm{P}\right]$ ATP (NEN) /Sambrook et al. 1989 .

\section{Reaction conditions}

Standard reaction conditions consisted of $25 \mathrm{~mm}$ Tris acetate (pH 7.5), $1 \mathrm{~mm}$ dithiothreitol, $1 \mathrm{~mm}$ phosphoenolpyruvate, 4 $\mathrm{U} / \mathrm{ml}$ of pyruvate kinase, $10 \mu \mathrm{M}$ nucleotides linear dsDNA /1.15 nM dsDNA molecules), $2 \mu \mathrm{M}$ SSB protein, $0.31 \mathrm{nM}$ total RecBCD enzyme (0.115 nM functional), $2 \mathrm{~mm}$ magnesium acetate $(\mathrm{pH}$ 7.51 , and $1 \mathrm{~mm}$ ATP. In some cases, RecBCD enzyme was varied as indicated. Assays were performed at $37^{\circ} \mathrm{C}$ and were initiated with the addition of enzyme after preincubation of all other components for $3 \mathrm{~min}$.

\section{Analysis of reaction products}

After a 4-min incubation with the RecBCD enzyme, each reaction $(40 \mu l)$ was stopped by the addition of $10 \mu \mathrm{l}$ of stop buffer (0.1 M EDTA, $2.5 \%$ SDS, 40\% glycerol, $0.125 \%$ bromophenol blue, $0.125 \%$ xylene cyanol). Samples were subject to electrophoresis through $1 \%$ native agarose gels for $15 \mathrm{hr}$ at $1.4 \mathrm{~V} / \mathrm{cm}$ in TAE (40 mM Tris-acetate at pH 8.0, 2 mM EDTA). The gels were dried and exposed to Kodak XAR-5 film at room temperature. Samples $(40 \mu \mathrm{l})$, which were analyzed by denaturing alkaline agarose electrophoresis, were stopped, and $10 \mu \mathrm{l}$ of alkaline loading buffer was added $(300 \mathrm{~mm} \mathrm{NaOH}, 6 \mathrm{~mm}$ EDTA, $18 \%$ Ficoll, $0.15 \%$ bromocresol green, $0.25 \%$ xylene cyanol). Except for Figure 5, all reactions without RecBCD enzyme were onehalf volume of those with RecBCD enzyme. The samples were mixed and then subjected to electrophoresis through $1 \%$ alkaline agarose gels for $15 \mathrm{hr}$ at $1.4 \mathrm{~V} / \mathrm{cm}$ in alkaline electrophoresis buffer (50 mM NaOH, $1 \mathrm{~mm}$ EDTA)

\section{Formation of ssDNA-tailed substrates using Exo III}

Plasmid pBR322 $\chi^{+} \mathrm{F}$ DNA (10 $\left.\mu \mathrm{g}\right)$, which was linearized with HindIII, was incubated with 1750 units of Exo III using reaction conditions according to Promega $166 \mathrm{~mm}$ Tris- $\mathrm{HCl}$ at $\mathrm{pH} 8.0$, $0.66 \mathrm{mM} \mathrm{MgCl}_{2}$ ) at $22^{\circ} \mathrm{C}$ for $1 \mathrm{~min}$. The reactions were stopped by the addition of $20 \mathrm{~mm}$ EDTA (pH 8.0 ) and $300 \mathrm{~mm} \mathrm{NaCl}$, heat treated at $75^{\circ} \mathrm{C}$ for $15 \mathrm{~min}$ and phenol/chloroform extracted as described in Sambrook et al. (1989). Control reactions were performed by adding Exo III to the DNA after the addition of stop buffer. The DNA was then precipitated by the addition of 2 volumes of $100 \%$ ethanol, washed twice with $70 \%$ ethanol, and resuspended in $100 \mu \mathrm{l}$ of TE buffer. The tailed DNA and the control DNA were then either digested with AseI to remove the ssDNA tail upstream from $\chi$, or incubated in restriction buffer without AseI. This DNA was then $5^{\prime}$-end-labeled as described previously, treated with varying total RecBCD enzyme concentrations at either $0 \mathrm{~mm}, 0.37 \mathrm{nM}$, or $37 \mathrm{nM}(0 ; 1$ functional RecBCD enzyme per 17 dsDNA ends; or 6 functional RecBCD enzymes per dsDNA end for the DNA substrates that were not cut with AseI) under optimal conditions and analyzed as described previously.

\section{Acknowledgments}

We are grateful to Frank Stahl for helpful discussions about these results. We thank the members of this laboratory for their critical reading of the manuscript. This work was supported by the funds from the National Institutes of Health grant (GM41347).

The publication costs of this article were defrayed in part by payment of page charges. This article must therefore be hereby marked "advertisement" in accordance with 18 USC section 1734 solely to indicate this fact.

\section{References}

Alatossava, T., H. Jutte, A. Kuhn, and E. Kellenberger. 1985. Manipulation of intracellular magnesium content in polymyxin B nonapeptide-sensitized Escherichia coli by ionophore A23187. J. Bacteriol. 162: 413-419.

Anderson, D.G., J.J. Churchill, and S.C. Kowalczykowski. 1997. Chi-activated RecBCD enzyme possesses 5' to 3' nucleolytic activity, but RecBC enzyme does not: Evidence suggesting that the alteration induced by Chi is not simply ejection of the RecD subunit. Genes to Cells. (in press).

Barbour, S.D., H. Nagaishi, A. Templin, and A.J. Clark. 1970. Biochemical and genetic studies of recombination proficiency in Escherichia coli. II. $\mathrm{rec}^{+}$revertants caused by indirect suppression of $\mathrm{rec}^{-}$mutations. Proc. Natl. Acad. Sci. 67: 128-135.

Cheng, K.C. and G.R. Smith. 1989. Distribution of Chi-stimulated recombinational exchanges and heteroduplex endpoints in phage lambda. Genetics 123: 5-17.

Clark, A.J. and A.D. Margulies. 1965. Isolation and characterization of recombination-deficient mutants of Escherichia coli K12. Proc. Nat1. Acad. Sci. 53: 451-459.

Dixon, D.A. and S.C. Kowalczykowski. 1991. Homologous pairing in vitro stimulated by the recombination hotspot, Chi. Cell 66: 361-371.

- 1993 . The recombination hotspot, Chi, is a regulatory sequence that acts by attenuating the nuclease activity of the Escherichia coli RecBCD enzyme. Cell 73: 87-96.

1995. Role of the Escherichia coli recombination hotspot, chi, in RecABCD-dependent homologous pairing. $J$. Biol. Chem. 270: 16360-16370.

Dixon, D.A., J.J. Churchill, and S.C. Kowalczykowski. 1994. Reversible inactivation of the Escherichia coli RecBCD enzyme by the recombination hotspot, Chi, in vitro: Evidence for functional inactivation or loss of the RecD subunit. Proc. Natl. Acad. Sci. 91: 2980-2984.

Eggleston, A. K. and S.C. Kowalczykowski. 1993. Biochemical characterization of a mutant RecBCD enzyme, the $\mathrm{RecB}^{2109} \mathrm{CD}$ enzyme, which lacks $\chi$-specific, but not nonspecific, nuclease activity. J. Mol. Biol. 231: 605-620.

Eichler, D.C. and I.R. Lehman. 1977. On the role of ATP in phosphodiester bond hydrolysis catalyzed by the recBC deoxyribonuclease of Escherichia coli. J. Biol. Chem. 252: 499503.

Emmerson, P.T. 1968. Recombination deficient mutants of Escherichia coli $\mathrm{K} 12$ that map between thyA and $\arg A$. Genetics 60: 19-30.

Ennis, D.G., S.K. Amundsen, and G.R. Smith. 1987. Genetic functions promoting homologous recombination in Escherichia coli: A study of inversions in phage lambda. Genetics 115: 11-24. 
Gillen, J.R., A.E. Karu, H. Nagaishi, and A.J. Clark. 1977. Characterization of the deoxyribonuclease determined by lambda reverse as exonuclease VIII of Escherichia coli. J. Mol. Biol. 113: $27-41$.

Howard-Flanders, P. and L. Theriot. 1966. Mutants of Escherichia coli $\mathrm{K}-12$ defective in DNA repair and in genetic recombination. Genetics 53: 1137-1150.

Joseph, J.W. and R. Kolodner. 1983a. Exonuclease VIII of Escherichia coli. I. Purification and physical properties. I. Biol. Chem. 258: 10411-10417.

- 1983 b. Exonuclease VIII of Escherichia coli. II. Mechanism of action. I. Biol. Chem. 258: 10418-10424.

Kogoma, T. 1996. Recombination by replication. Cell 85: 625627.

Konforti, B.B. and R.W. Davis. 1987. 3' homologous free ends are required for stable joint molecule formation by the RecA and single-stranded binding proteins of Escherichia coli. Proc. Natl. Acad. Sci. 84: 690-694.

- 1990. The preference for a 3' homologous end is intrinsic to RecA-promoted strand exchange. I. Biol. Chem. 265: 6916-6920.

Koppen, A., S. Krobitsch, B. Thoms, and W. Wackernagel. 1995. Interaction with the recombination hot spot Chi in vivo converts the RecBCD enzyme of Escherichia coli into a Chiindependent recombinase by inactivation of the RecD subunit. Proc. Natl. Acad. Sci. 92: 6249-6253.

Korangy, F. and D.A. Julin. 1993. Kinetics and processivity of ATP hydrolysis and DNA unwinding by the recBC enzyme from Escherichia coli. Biochemistry 32: 4873-4880.

Kowalczykowski, S.C. and A.K. Eggleston. 1994. Homologous pairing and DNA strand-exchange proteins. Annu. Rev. Biochem. 63: 991-1043.

Kowalczykowski, S.C., D.A. Dixon, A.K. Eggleston, S.D. Lauder, and W. Rehrauer. 1994. Biochemistry of homologous recombination in Escherichia coli. Microbiol. Rev. 58: 401465.

Lam, S.T., M.M. Stahl, K.D. McMilin, and F.W. Stahl. 1974. Rec-mediated recombinational hot spot activity in bacteriophage lambda. II. A mutation which causes hot spot activity. Genetics 77: 425-433.

LeBowitz, J. 1985. "Biochemical mechanism of strand initiation in bacteriophage lambda DNA replication." $\mathrm{PhD}$ thesis, John Hopkins University, Baltimore, MD.

Lovett, S.T., C. Luisi-DeLuca, and R.D. Kolodner. 1988. The genetic dependence of recombination in $r e c D$ mutants of Escherichia coli. Genetics 120: 37-45.

Masterson, C., P.E. Boehmer, F. McDonald, S. Chaudhuri, I.D. Hickson, and P.T. Emmerson. 1992. Reconstitution of the activities of the RecBCD holoenzyme of Escherichia coli from the purified subunits. J. Biol. Chem. 267: 13564-13572.

McEntee, K., G.M. Weinstock, and I.R. Lehman. 1980. RecA protein-catalyzed strand assimilation: Stimulation by Escherichia coli single-stranded DNA-binding protein. Proc. Natl. Acad. Sci. 77: 857-861.

Myers, R.S., A. Kuzminov, and F.W. Stahl. 1995a. The recombination hot spot Chi activates RecBCD recombination by converting Escherichia coli to a recD mutant phenocopy. Proc. Natl. Acad. Sci. 92: 6244-6268.

Myers, R.S., M.M. Stahl, and F.W. Stahl. 1995b. Chi recombination activity in phage lambda decays as a function of genetic distance. Genetics 141: 805-812.

Palas, K.M. and S.R. Kushner. 1990. Biochemical and physical characterization of exonuclease $\mathrm{V}$ from Escherichia coli. Comparison of the catalytic activities of the RecBC and RecBCD enzymes. I. Biol. Chem. 265: 3447-3454.

Ponticelli, A.S., D.W. Schultz, A.F. Taylor, and G.R. Smith.
1985. Chi-dependent DNA strand cleavage by recBC enzyme. Cell 41: 145-151.

Register, J.C. III and J. Griffith. 1985. The direction of RecA protein assembly onto single strand DNA is the same as the direction of strand assimilation during strand exchange. $J$. Biol. Chem. 260: 12308-12312.

Resnick, M.A. 1976. The repair of double-strand breaks in DNA: A model involving recombination. J. Theor. Biol. 59: 97-106.

Rinken, R., B. Thomas, and W. Wackernagel. 1992. Evidence that recBC-dependent degradation of duplex DNA in Escherichia coli recD mutants involves DNA unwinding. J. Bacteriol. 174: 5424-5429.

Roman, L.J. and S.C. Kowalczykowski. 1989a. Characterization of the helicase activity of the Escherichia coli RecBCD enzyme using a novel helicase assay. Biochemistry 28: 2863 2873.

1989b. Formation of heteroduplex DNA promoted by the combined activities of Escherichia coli recA and recBCD proteins. J. Biol. Chem. 264: 18340-18348.

Roman, L.J., A.K. Eggleston, and S.C. Kowalczykowski. 1992. Processivity of the DNA helicase activity of Escherichia coli RecBCD enzyme. I. Biol. Chem. 267: 4207-4214.

Ruyechan, W.T. and J.G. Wetmur. 1975. Studies on the cooperative binding of the Escherichia coli DNA unwinding protein to single-stranded DNA. Biochemistry 14: 5529-5534.

Sambrook, J., E.F. Fritsch, and T. Maniatis. 1989. Molecular cloning: A laboratory manual, 2nd ed. Cold Spring Harbor Laboratory Press, Cold Spring Harbor, NY.

Shibata, T., R.P. Cunningham, C. DasGupta, and C.M. Radding. 1979. Homologous pairing in genetic recombination: Complexes of recA protein and DNA. Proc. Nat1. Acad. Sci. 76: $5100-5104$.

Smith, G.R. 1988. Homologous recombination in procaryotes. Microbiol. Rev. 52: 1-28.

-1990. RecBCD enzyme. In Nucleic acids and molecular biology (ed. F. Eckstein and D.M.J. Lilley), pp. 78-98. Springer-Verlag, Berlin.

Smith, G.R., S.M. Kunes, D.W. Schultz, A. Taylor, and K.L. Triman. 1981. Structure of Chi hotspots of generalized recombination. Cell 24: 429-436.

Stahl, F.W., J.M. Crasemann, and M.M. Stahl. 1975. Rec-mediated recombinational hot spot activity in bacteriophage lambda. III. Chi mutations are site-mutations stimulating rec-mediated recombination. J. Mol. Biol. 94: 203-212.

Stahl, F.W., M.M. Stahl, R.E. Malone, and J.M. Crasemann. 1980. Directionality and nonreciprocality of Chi-stimulated recombination in phage lambda. Genetics 94: 235-248.

Stahl, F.W., L.C. Thomason, I. Siddiqi, and M.M. Stahl. 1990. Further tests of a recombination model in which Chi removes the RecD subunit from the RecBCD enzyme of Escherichia coli. Genetics 126: 519-533.

Sun, H., D. Treco, and J.W. Szostak. 1991. Extensive 3'-overhanging, single-stranded DNA associated with the meiosisspecific double-strand breaks at the ARG4 recombination initiation site. Cell 64: 1155-1161.

Szostak, J.W., T.L. Orr-Weaver, R.J. Rothstein, and F.W. Stahl. 1983. The double-strand break repair model for recombination. Cell 33: 25-35.

Taylor, A.F. 1988. The recBCD enzyme of Escherichia coli. In Genetic recombination (ed. R. Kucherlapati and G.R. Smith), pp. 231-263. American Society for Microbiology, Washington, D.C.

Taylor, A.F. and G.R. Smith. 1985. Substrate specificity of the DNA unwinding activity of the RecBC enzyme of Escherichia coli. J. Mol. Biol. 185: 431-443.

1995. Strand specificity of nicking of DNA at Chi sites 
by RecBCD enzyme. Modulation by ATP and magnesium levels. J. Biol. Chem. 270: 24459-24467.

Taylor, A.F., D.W. Schultz, A.S. Ponticelli, and G.R. Smith. 1985. RecBC enzyme nicking at Chi sites during DNA unwinding: Location and orientation-dependence of the cutting. Cell 41: 153-163.

Telander-Muskavitch, K.M. and S. Linn. 1981. RecBC-like enzymes: Exonuclease V deoxyribonucleases. In The enzymes (ed. P.D. Boyer), pp. 233-250. Academic Press, NY.

Templin, A., S.R. Kushner, and A.J. Clark. 1972. Genetic analysis of mutations indirectly suppressing $r e c B$ and $r e c C$ mutations. Genetics 72: 205-215.

Thaler, D.S., E. Sampson, I. Siddiqi, S.M. Rosenberg, F.W. Stahl, and M. Stahl. 1988. A hypothesis: Chi-activation of recBCD enzyme involves removal of the recD subunit. In Mechanisms and consequences of DNA damage processing (ed. E. Friedberg and P. Hanawalt), pp. 413-422. Alan R. Liss, NY.

Tracy, R.B. and S.C. Kowalczykowski. 1996. In vitro selection of preferred DNA pairing sequences by the Escherichia coli RecA protein. Genes \& Dev. 10: 1890-1903.

White, C.I. and J.E. Haber. 1990. Intermediates of recombination during mating type switching in Saccharomyces cerevisiae. $E M B O$ J. 9: 663-673. 


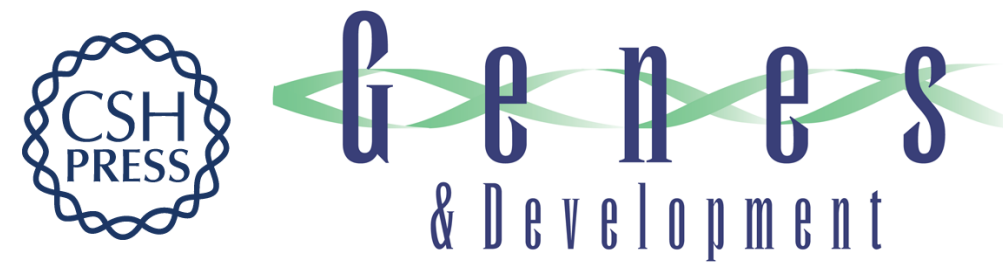

\section{The recombination hot spot chi is a regulatory element that switches the polarity of DNA degradation by the RecBCD enzyme.}

D G Anderson and S C Kowalczykowski

Genes Dev. 1997, 11:

Access the most recent version at doi:10.1101/gad.11.5.571

References This article cites 52 articles, 33 of which can be accessed free at: http://genesdev.cshlp.org/content/11/5/571.full.htmI\#ref-list-1

License

Email Alerting Receive free email alerts when new articles cite this article - sign up in the box at the top Service right corner of the article or click here.

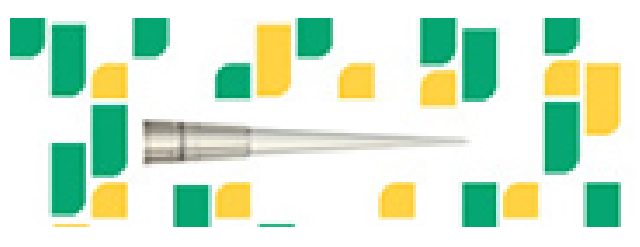

Focused on your science. 